\title{
Sound propagation over screened ground under upwind conditions
}

\section{Rasmussen, Karsten Bo}

\section{Published in:}

Acoustical Society of America. Journal

Link to article, DOI:

10.1121/1.417223

Publication date:

1996

Document Version

Publisher's PDF, also known as Version of record

Link back to DTU Orbit

Citation (APA):

Rasmussen, K. B. (1996). Sound propagation over screened ground under upwind conditions. Acoustical Society of America. Journal, 100(6), 3581-3586. https://doi.org/10.1121/1.417223

\section{General rights}

Copyright and moral rights for the publications made accessible in the public portal are retained by the authors and/or other copyright owners and it is a condition of accessing publications that users recognise and abide by the legal requirements associated with these rights.

- Users may download and print one copy of any publication from the public portal for the purpose of private study or research.

- You may not further distribute the material or use it for any profit-making activity or commercial gain

- You may freely distribute the URL identifying the publication in the public portal

If you believe that this document breaches copyright please contact us providing details, and we will remove access to the work immediately and investigate your claim. 


\title{
Sound propagation over screened ground under upwind conditions
}

\author{
Karsten Bo Rasmussen \\ Department of Acoustic Technology, Building 352, Technical University of Denmark, DK-2800 Lyngby, \\ Denmark
}

(Received 30 September 1995; revised 12 March 1996; accepted 22 July 1996)

\begin{abstract}
A screen on an absorbing ground is investigated experimentally and theoretically under upwind conditions. The experimental data are the result of scale model experiments in a 1:25 scale model. The sound propagation is measured using a triggered spark source with signal spectrum averaging in the frequency domain. The meteorological data representing the wind conditions have been determined by means of hot-wire anemometry in positions on both sides of the screen as well as directly over the screen. The theoretical model used for comparison is a hybrid approach. The sound field without a barrier is determined by means of numerical integration of a Hankel transform solution for a stratified atmosphere [like a fast field program (FFP) but taking the near field into account]. This solution is used on both sides of the screen and is combined with a screen diffraction calculation. Results from the calculation model are able to explain the overall tendency in the experimental results. (C) 1996 Acoustical Society of America.
\end{abstract}

PACS numbers: 43.28.Fp, 43.50.Vt [LCS]

\section{INTRODUCTION}

Sound barriers have been studied extensively and are widely used as a practical solution to many outdoor noise problems.

The theoretical description of the influence of a simple screen has reached an advanced level when the atmosphere is still and homogeneous. ${ }^{1-6}$ However, atmospheric effects play a very important part when it comes to sound propagation over screens, especially in the upwind case, where the screen is an efficient means of reducing the sound level. In the downwind case, different conditions exist since the influence of the screen is expected to be reduced by the presence of wind. This case has been studied theoretically by means of a parabolic equation (PE) method. ${ }^{7}$ An earlier attempt to perform scale model studies of acoustic barriers in the presence of wind has been reported by DeJong and Stusnick. ${ }^{8}$

The influence of wind speed fluctuationsturbulence-is ignored in this study, and average wind speeds are used throughout. Turbulence is a very important parameter over long distances ${ }^{9-12}$ and for high frequencies, and maybe even more so when a screen is present. When the wind speed decreases, so does the influence of turbulence. Experimental evidence suggests that for the moderate wind speeds studied in this work turbulence plays a secondary role only.

In Sec. I a new theoretical concept for calculation of approximated results for outdoor propagation over screened ground is described. The concept is based on a combination of theory for propagation in a stratified atmosphere having variations in effective sound speed, and theory for diffraction by a screen in a still homogeneous atmosphere. Hence, the calculation model takes the diffraction process at the screen into account in an accurate way, but ignoring the wind, and it takes the wind into account in an accurate way but ignoring the screen. The model is faster than a PE-type calculation.

In Sec. II comparisons with results from scale model experiments are made. Data are obtained in a scale model using a 1:25 scaling ratio. A triggered spark source described previously in more detail ${ }^{5}$ is placed in a small open ended boundary layer wind tunnel which is $0.8 \mathrm{~m}$ wide, $0.37 \mathrm{~m}$ high, and $5.4 \mathrm{~m}$ long. The wind speed was generated by a low-noise axial fan located at the exhaust end. The tunnel walls and ceiling were actually lowered onto the measuring surface previously used for measurements without wind ${ }^{5}$ (see Fig. 1). A high precision Streamline measuring system ${ }^{13}$ with single wire 55p11 anemometer probes was used in various positions in the tunnel. Roughness elements were placed at the intake of the tunnel in order to help creating a reasonable wind speed profile. No temperature gradient was present in the tunnel.

The acoustic receiver was a 1/8-in. 4138 Brüel \& Kjaer microphone located as far away from the tunnel walls as possible and oriented for grazing sound incidence on the diaphragm. The acoustic results are obtained from frequency domain averaging on a power basis of propagation of sound from a spark source. In previous work ${ }^{5}$ time domain averaging was used and the improvement of signal-to-noise ratio associated with this technique was exploited, but this is not possible when a turbulent flow is present due to the modulation of the transfer function associated with the turbulence. Time domain averaging is only permissible when the propagation process may be considered stationary.

The thermodynamic boundary layer effect has been ignored in this work since an earlier investigation ${ }^{14}$ revealed it had very little influence on this effect at the moderate scaling ratio of 1:25 used here.

The scale modeling technique makes it possible to obtain results under carefully controlled atmospheric conditions, something which is hard to obtain under full scale conditions. 
Inlet

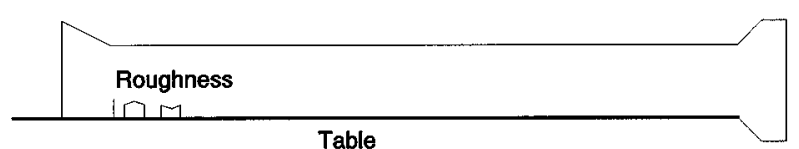

FIG. 1. Sketch of wind tunnel arrangement.

\section{THEORY}

This paper is based on diffraction theory originating from Kouyoumjian and Pathak ${ }^{15,16}$ for a simple screen in a stationary homogeneous atmosphere. This theory is reinterpreted to form an approximate solution in a moving atmosphere. To this end, a full wave solution based on a Hankel transform ${ }^{17,18}$ is used for a point source in a layered atmosphere over an impedance surface. The results from this solution, which are found from numerical integration, are interpreted as a direct ray and a reflected ray with an appropriate complex reflection factor. In spite of the windy conditions, the waves are assumed to follow straight lines. Approaches where straight line propagation is used, even when wind is present, have previously been found to be successful ${ }^{19}$ when the source height used in the calculations is adjusted in accordance with the basic refraction caused by the wind. The ray interpretation of these results is then used in conjunction with the screen diffraction approach, both on the source as well as on the receiver side of the screen. In this way an approximate solution to screen diffraction in the presence of wind is obtained. However, it must be emphasized that only the laminar flow is taken into account. The turbulent eddies formed at the screen are not taken into account.

\section{A. Theory for screen diffraction}

A previously published theory ${ }^{16}$ was used for calculation of the diffraction process at the screen. The theory is based on the uniform theory of diffraction ${ }^{5,15}$ (UTD), in combination with a ray interpretation of sound propagation over finite impedance ground.

The UTD calculations are based on the expressions for diffraction by a wedge-shaped barrier of infinite impedance. The diffracted sound pressure for the special case of a thin screen is (the sound rays being normal to the edge and using $e^{j \omega t}$ notation)

$p=\frac{e^{-j k R_{1}}}{R_{1}}\left[V\left(r_{0} r_{1} / R_{1}, \theta_{1}-\theta_{0}\right)+V\left(r_{0} r_{1} / R_{1}, \theta_{1}+\theta_{0}\right)\right]$.

Here, $k$ is the wave number and the geometrical parameters are defined in Fig. 2. Furthermore, one has the following definitions:

$$
\begin{aligned}
& V(A, \theta)=V^{+}(A, \theta)+V^{-}(A, \theta), \\
& V^{ \pm}(A, \theta)=\frac{-e^{-j \pi / 4}}{\sqrt{2 \pi k A}} \frac{1}{4} \cot \left(\frac{\pi \pm \theta}{4}\right) F^{\prime}\left(X^{ \pm}(\theta)\right), \\
& X^{ \pm}(\theta)=2 k A \cos ^{2}(\theta / 2),
\end{aligned}
$$

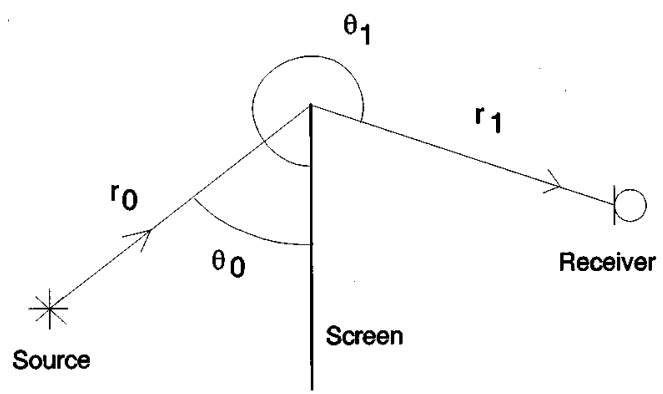

FIG. 2. Geometrical parameters at screen. $R_{1}=r_{0}+r_{1}$.

$$
F^{\prime}(z)=2 j \sqrt{z} e^{j z} F(\sqrt{z}),
$$

where $F$ is the Fresnel integral,

$$
F(\sqrt{z})=\int_{\sqrt{z}}^{\infty} \exp \left(-j t^{2}\right) d t .
$$

Similar but more general equations valid for a wedge-shaped object, such as an earth berm, may be found in previous work. $^{5,12}$

\section{B. Theory for propagation over unobstructed terrain}

The field over unobstructed terrain but taking the influence of wind into account is found from the inverse Hankel transform in pressure, ${ }^{18}$

$$
p(r, z)=\int_{0}^{\infty} K_{p}(s, z) J_{0}(s r) s d s,
$$

where $s$ is the horizontal wave number component. For isovelocity layers the kernel $K_{p}$ is given by

$$
K_{p}(s, z)=A^{-}(s) e^{-z \alpha(s)}+A^{+}(s) e^{z \alpha(s)},
$$

where $\alpha(s)$ is $\left(s^{2}-k^{2}\right)^{1 / 2}$. Here, $A^{-}$and $A^{+}$are determined numerically according to the boundary conditions.

For gradient layers Airy functions are introduced as follows:

$$
K_{p}(s, z)=A^{-}(s) V(\tau+y)+A^{+}(s) W+(\tau+y),
$$

where

$$
\tau=\left(s^{2}-k_{0}^{2}\right) n^{2}, \quad y=z / n, \quad n=\operatorname{sign}(\gamma)\left(|\gamma| 2 k_{0}^{2}\right)^{-1 / 3},
$$

$k_{0}$ being the wave number at ground level and

$$
V(z)=\pi^{1 / 2} \operatorname{Ai}(z), \quad W(z)=2 \pi^{1 / 2} e^{j \pi / 6} \operatorname{Ai}\left(z e^{j 2 \pi / 3}\right) .
$$

Ai denotes the Airy function and the velocity gradient $\gamma$ is defined so that

$$
k(z)=\omega /\left(c_{0}[1+\gamma z]\right),
$$

where $z$ is height over ground and $c_{0}$ is sound speed at ground level.

A set of similar relations valid for the vertical displacement component (air particle displacement) is necessary in order to obtain a set of linear equations characterizing a layered atmospheric structure. When a monopole point source field is introduced on the right-hand side of the equations for 
the kernels, and when a impedance condition is introduced at ground level, the set of linear equations becomes complete and can be solved by conventional Gauss-elimination techniques. The details are found in the original references. ${ }^{17,18}$ The approach outlined here is similar to the well-known fast field program approaches, ${ }^{20}$ except for the inclusion of the near field in the approach used in this work.

The impedance model used in this work is the twoparameter model described by Attenborough ${ }^{21}$ as the $2 \mathrm{PA}$ model. In the present notation $\left(e^{j \omega t}\right)$ it reads thus,

$$
Z(\sigma, \beta)=\sqrt{\frac{\sigma}{f}} 0.4342(1-j)-j \frac{\beta}{f} 9.6485,
$$

where standard values (for $20^{\circ} \mathrm{C}$ ) have been inserted for sound speed $(340 \mathrm{~m} / \mathrm{s})$, density $\left(1.20413 \mathrm{~kg} / \mathrm{m}^{3}\right)$, and ratio of specific heats (1.4021). Here, $Z$ denotes the relative characteristic impedance and $\beta$ denotes the rate of exponential decrease of porosity with depth. Alternatively, $\beta$ may be interpreted as $2 / d_{e}$, where $d_{e}$ is the effective thickness of a porous layer of constant porosity on a hard backing.

\section{Implementation}

The hybrid approach used for propagation over a screened terrain is based on a combination of the methods outlined in Secs. I A and I B. The field obtained from numerical integration according to Sec. I B is interpreted as a direct plus ground reflected field,

$$
p=\frac{e^{-j k R_{1}}}{R_{1}}+Q \frac{e^{-j k R_{2}}}{R_{2}},
$$

where $R_{1}$ is the direct path from source to receiver and $R_{2}$ is the reflected path from source to receiver. $Q$ denotes the spherical reflection factor characterized by amplitude, as well as phase. The distances $R_{1}$ and $R_{2}$ are determined from the geometry and $Q$ is then found from the equality between Eqs. (14) and (7). This procedure is carried out on the source side of the screen, as well as on the receiver side of the screen. The screen diffraction is determined by the formulas in Sec. I A. Only cases where no direct wave from source to receiver exist in the sense of geometrical optics have been considered in this work.

\section{RESULTS}

\section{A. Meteorological data from scale model}

The meteorological data are obtained from Dantec Streamline hot-wire anemometry equipment (using a type $55 \mathrm{p} 11$ single wire probe). The wind speed is found as an average over $54 \mathrm{~s}$. A meteorological sampling rate of $300 \mathrm{~Hz}$ was used; this rate was found to be more than sufficient. The anemometer probes were oriented with a vertical wire to ensure that the measurement was insensitive to vertical flow.

The wind speed profile was measured in positions (converted to full scale) $30 \mathrm{~m}$ before the screen; another profile measurement was made just above the screen and in positions $10 \mathrm{~m}$ after the screen. The results obtained are shown in Fig. 3. Note that the speed above the screen is higher than before and after the screen as a consequence of the redirection of the flow caused by the screen.

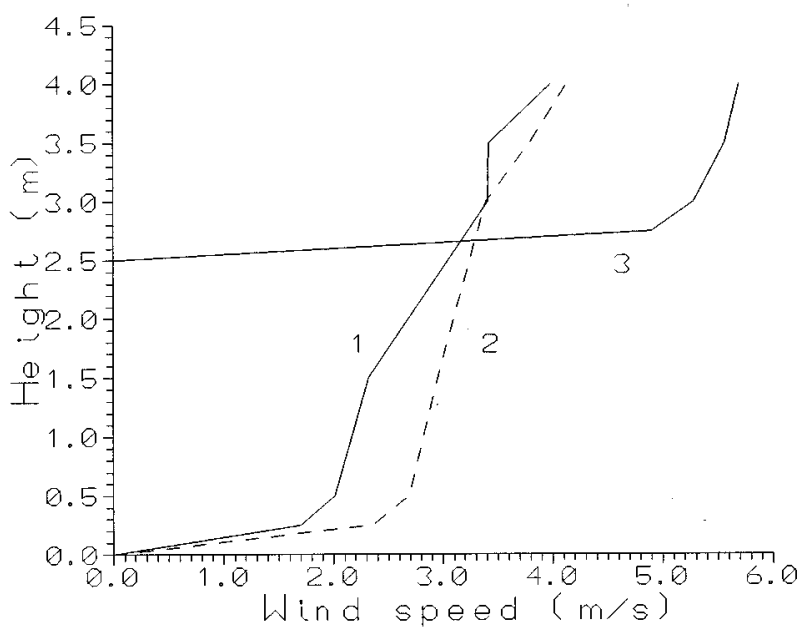

FIG. 3. Wind speed profiles for full scale heights. Curve 1 is measured on the source side of the screen, curve 2 on the receiver side, and curve 3 above screen.

Figure 4 shows another profile which is valid for unobstructed terrain when measurements are made halfway between source and receiver. This profile is steeper close to the ground than most full scale outdoor wind profiles. When a screen is present it serves as an additional roughness element, improving the shape of the profile.

\section{B. Comparison with acoustical data from scale model}

The acoustic measurements are based on energy averaging in the frequency domain of 20 pulses, each of which has been edited in the time domain so that reflections from tunnel walls, etc., are removed. The energy averaging is necessary because of the stochastic process involved in propagation under the influence of wind, which also means that the signal-to-noise ratio is potentially poor. Figure 5 shows the shape of the source spectrum (using model scale frequencies)

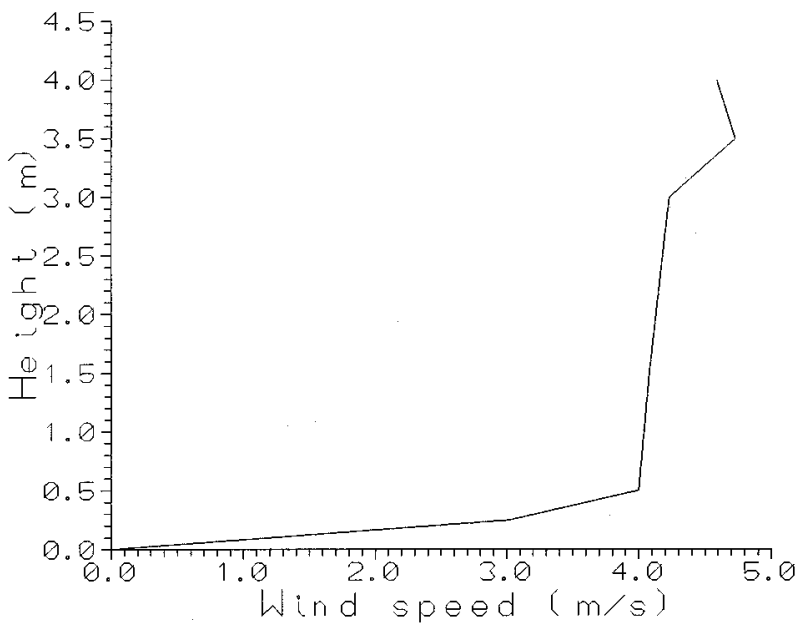

FIG. 4. Wind speed profile for full scale heights. Profile is valid for the case of unobstructed terrain. 


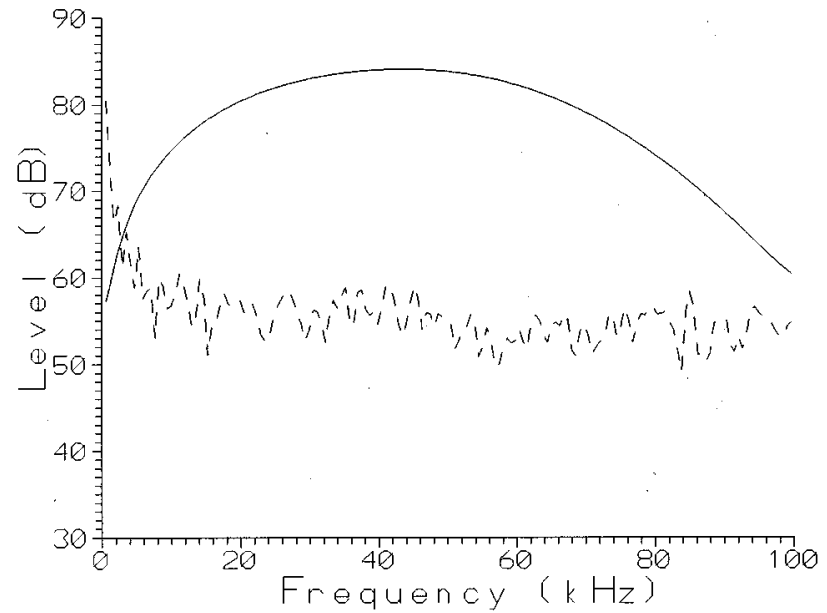

FIG. 5. Examples of measured ultrasonic data representing source spectrum, straight line, and noise spectrum in the presence of wind, dashed line.

in free field as well as a typical noise spectrum measured in the presence of wind. However, it should be remembered that the level at which these spectra occur depends on the situation investigated. The noise stems from electrical sources, except for lower frequencies where the wind generates additional noise when interacting with the microphone (flow noise). The frequency range of the measured data is limited to between 400 and $3000 \mathrm{~Hz}$ full scale for the screened cases investigated; i.e., the frequency range where the signal-to-noise ratio was satisfactory. This full scale frequency range corresponds to model scale frequencies between 10 and $75 \mathrm{kHz}$. Under the described measurement conditions, measurements could be repeated with deviations of the order $\pm 2 \mathrm{~dB}$. The basic setup involving the screen is shown in Fig. 6. The width of the screen was $9 \mathrm{~mm}$ corresponding to $22.5 \mathrm{~cm}$ in full scale. The $2 \mathrm{PA}$ impedance model, Eq. (13), was used to describe the acoustic impedance of the ground surface (which was a layer of thin cotton material on hard backing) with $\sigma=20 \mathrm{kN} \mathrm{s} \mathrm{m}^{-4}$ and $\beta=120$ $\mathrm{m}^{-1}$ for full scale frequencies. The value of $\beta$ used corresponds to a homogeneous porous layer of thickness $d_{e}=2 / \beta=0.0167 \mathrm{~m}$ on a hard backing. This full scale thickness is approximately 25 times the physical thickness of the material used in the scale model. The choice of parameter values in the 2PA model is the result of curve-fitting soundpressure level results as a function of full scale frequency for the case without wind and for unobstructed terrain. Figure 7 shows results for an unscreened case with a wind speed profile, as shown in Fig. 4. The agreement between measured data (dashed line) and calculated values (solid line) is seen to be very good. The theoretical curves are calculated as described in Sec. I B. (Results without wind and measured over

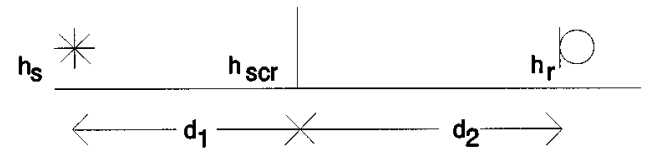

FIG. 6. Heights and distances for propagation over screen.

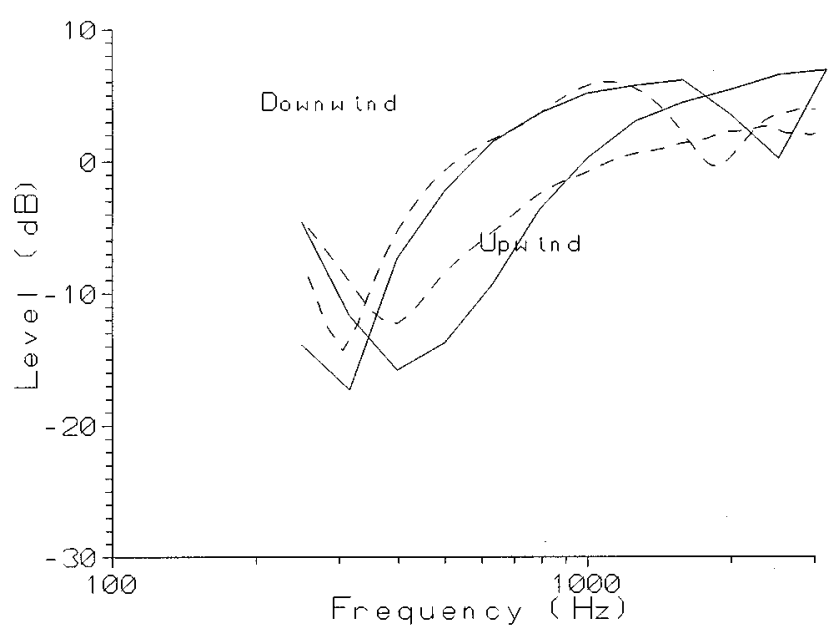

FIG. 7. Sound-pressure level $r e$ : free field. Full scale parameters: $h_{s}=2 \mathrm{~m}$, $h_{r}=2 \mathrm{~m}$, distance $80 \mathrm{~m}$. Straight line, calculated; dashed line, measured.

the same acoustic surface may be found in previous work, ${ }^{22}$ and they also agree very well with calculated data from the 2PA model.)

Figures 8-10 show results for different full scale geometries all involving the $2.5-\mathrm{m}$ high screen. The relevant wind speed profiles were shown in Fig. 3. For the case of no wind we see excellent agreement between measured and calculated data in Figs. 8 and 9. In Fig. 10 a signal-to-noise limitation may be limiting the depth of the minimum at $600 \mathrm{~Hz}$ in the upwind curve, since the noise level in the presence of wind was approximately $-28 \mathrm{~dB}$ at $600 \mathrm{~Hz}$. Nevertheless, the results generally agree well with calculations. However, in Fig. 8 the details in the interference pattern do not follow the calculated results. This is believed to be related to the fact that this case has the highest source and receiver positions.

Downwind simulations have also been tried, but with poor result. The problem seems to be that the wind carries the sound over the barrier in a fashion which is not taken into account in the model. Figure 11 displays results for the

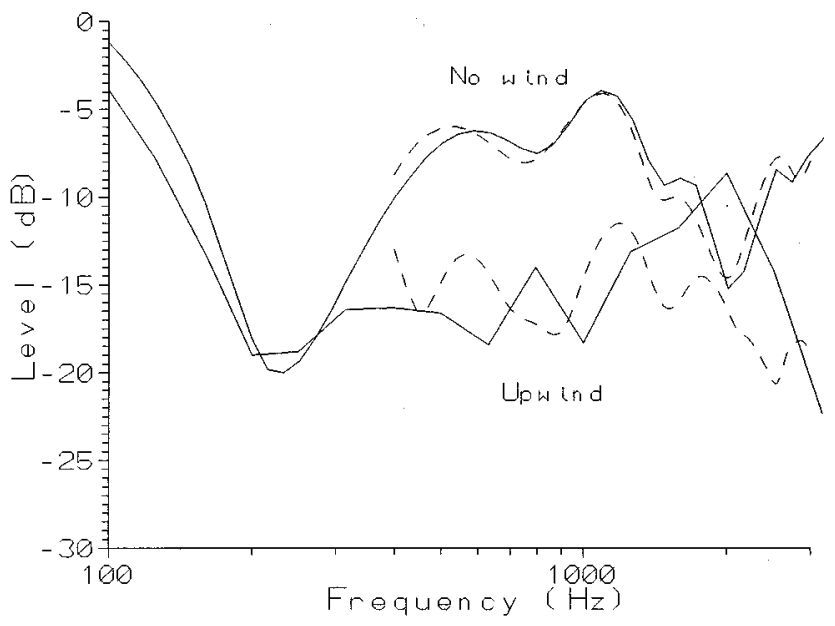

FIG. 8. Sound pressure-level $r e$ : free field. Full scale parameters: $h_{s}=2 \mathrm{~m}$, $h_{r}=2 \mathrm{~m}, d_{1}=60 \mathrm{~m}, d_{2}=20 \mathrm{~m}, h_{\mathrm{scr}}=2.5 \mathrm{~m}$. Straight line, calculated; dashed line, measured. 


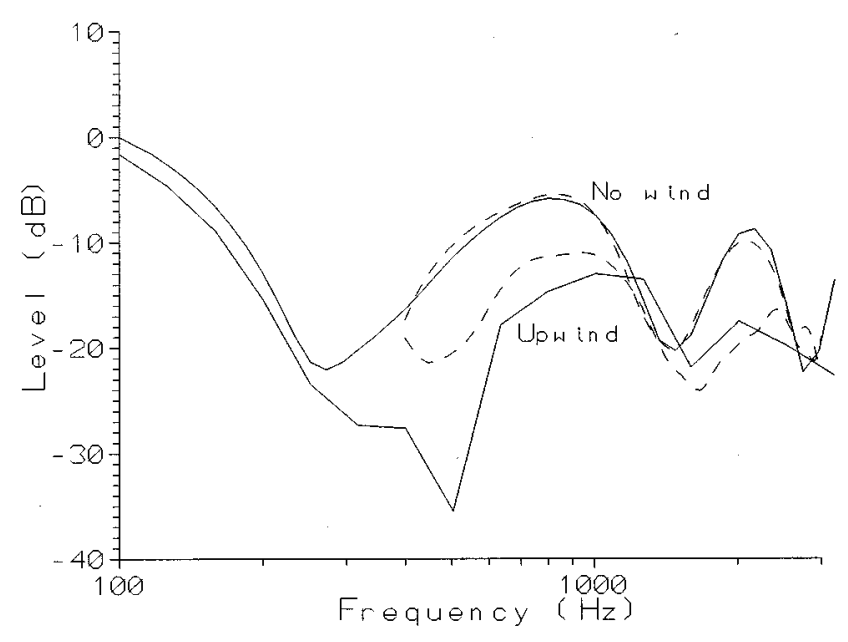

FIG. 9. Sound-pressure level $r e$ : free field. Full scale parameters: $h_{s}=2 \mathrm{~m}$, $h_{r}=1 \mathrm{~m}, d_{1}=40 \mathrm{~m}, d_{2}=20 \mathrm{~m}, h_{\mathrm{scr}}=2.5 \mathrm{~m}$. Straight line, calculated; dashed line, measured.

downwind case. A theoretical curve is included in the figure based on calculations ignoring the screen but taking into account the profile which is measured at the screen position. At heights lower than $2.5 \mathrm{~m}$ (full scale) the wind speed is set to zero due to the presence of the screen at these heights. The resulting profile is used for predictions and referred to as the modified profile. The comparison shows that by including just the profile and ignoring the screen, a first-order approximation to the measured result may be obtained. Hence, the mere profile could be as important as the screen itself in the downwind case.

\section{CONCLUSIONS}

A new hybrid model has been developed for the case of upwind propagation over ground in the shadow zone of a screen. The model is able to explain the overall tendency of the experimental results.

The model may be seen as a supplement to other calculation models, such as PE-type programs. In its present form

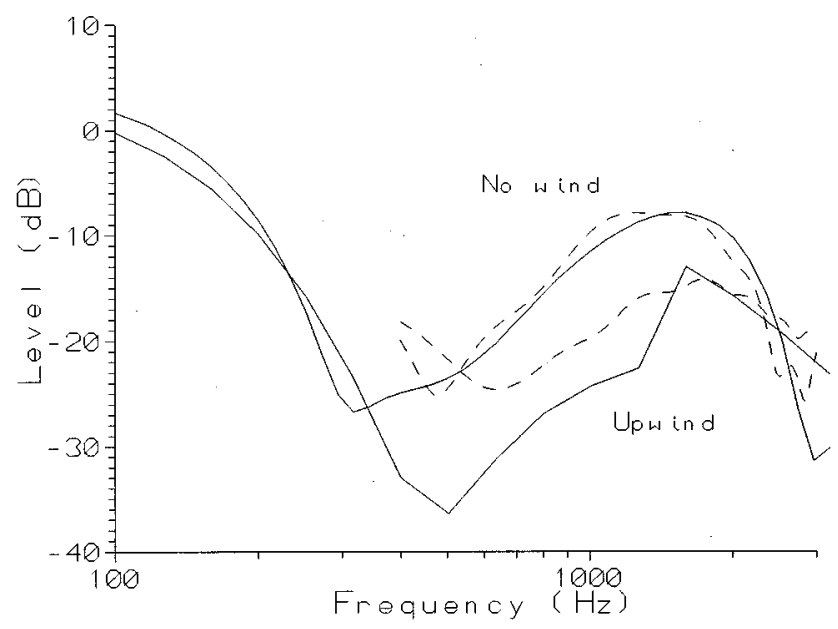

FIG. 10. Sound-pressure level re: free field. Full scale parameters: $h_{s}=1.35$ $\mathrm{m}, h_{r}=0.5 \mathrm{~m}, d_{1}=60 \mathrm{~m}, d_{2}=20 \mathrm{~m}, h_{\mathrm{scr}}=2.5 \mathrm{~m}$. Straight line, calculated; dashed line, measured.

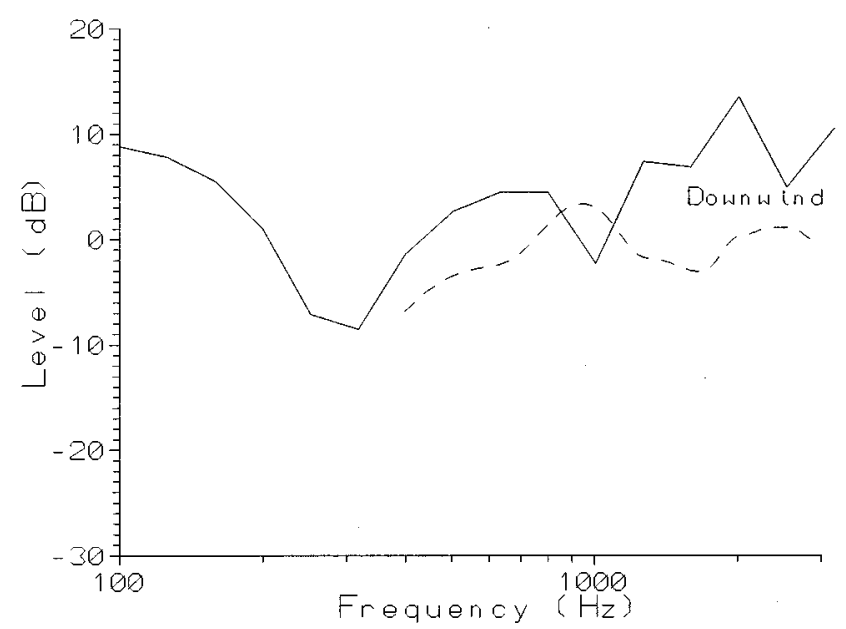

FIG. 11. Sound-pressure level re: free field. Full scale parameters: $h_{s}=2 \mathrm{~m}$, $h_{r}=1 \mathrm{~m}, d_{1}=20 \mathrm{~m}, d_{2}=40 \mathrm{~m}, h_{\mathrm{scr}}=2.5 \mathrm{~m}$. Straight line, calculated without taking screen into account but using modified profile; dashed line, measured.

the model is somewhat faster than the PE approach and future investigations shall reveal if the present approach can be developed into a truly fast model for engineering applications.

The model has been tested with a series of data from elaborate scale model experiments with carefully controlled atmospheric conditions, something which is hard to obtain under full scale conditions.

The model is only useful under upwind conditions. In a downwind situation the sound is refracted over the top of the barrier in a manner which cannot easily be taken into account in the model.

\section{ACKNOWLEDGMENT}

Financial support from H. C. Ørsteds Foundation is gratefully acknowledged.

${ }^{1}$ H. Medwin, "Shadowing by finite noise barriers," J. Acoust. Soc. Am. 69, 1060-1064 (1981).

${ }^{2}$ W. J. Hadden and A. D. Pierce, "Sound diffraction around screens and wedges for arbitrary source locations," J. Acoust. Soc. Am. 69, 12661276 (1981).

${ }^{3}$ D. J. Saunders and R. D. Ford, "A study of the reduction of explosive impulses by finite sized barriers," J. Acoust. Soc. Am. 94, 2859-2875 (1993).

${ }^{4}$ R. Raspet, J. Ezell, and S. V. Coggeshall, "Diffraction of an explosive transient," J. Acoust. Soc. Am. 79, 1326-1334 (1986).

${ }^{5}$ K. B. Rasmussen, "Model experiments related to outdoor sound propagation over an earth berm," J. Acoust. Soc. Am. 96, 3617-3620 (1994).

${ }^{6}$ Y. W. Lam and S. C. Roberts, "A simple method for accurate prediction of finite barrier insertion loss," J. Acoust. Soc. Am. 93, 1445-1452 (1993).

${ }^{7}$ E. M. Salomons, "Diffraction by a screen in downwind sound propagation: A parabolic-equation approach," J. Acoust. Soc. Am. 95, 31093117 (1994).

${ }^{8}$ R. DeJong and E. Stusnick, "Scale model studies of the effects of wind on acoustic barrier performance," J. Noise Control Eng. 6(3), 101-109 (1976).

${ }^{9}$ G. A. Daigle, "Effects of atmospheric turbulence on the interference of sound waves above a finite impedance boundary," J. Acoust. Soc. Am. 65, 45-49 (1979).

${ }^{10}$ M. R. Stinson, D. I. Havelock, and G. A. Daigle, "Comparison of predicted and measured sound pressure levels within a refractive shadow in the presence of turbulence," in Proceedings of Inter-Noise 95, 10-12 July 
1995, Newport Beach, CA, pp. 327-330.

${ }^{11}$ A. L'Espérance, Y. Gabillet, and G. A. Daigle, “'Outdoor sound propagation in the presence of atmospheric turbulence: Experiments and theoretical analysis with the fast field program algorithm," J. Acoust. Soc. Am. 98, 570-579 (1995).

${ }^{12} \mathrm{R}$. Raspet and W. Wu, "Calculation of average turbulence effects on sound propagation based on the fast field program formulation," J. Acoust. Soc. Am. 97, 147-153 (1995).

${ }^{13} \mathrm{~F}$. E. Jørgensen, “A new concept for an automated hot-wire anemometer with fully integrated experiment manager,' Dantec Information 13 (January 1994).

${ }^{14}$ K. B. Rasmussen, "'Sound propagation from a point source over a twoimpedance surface," Acta Acustica 2, 173-177 (1994).

${ }^{15}$ R. G. Kouyoumjian and P. H. Pathak, "A uniform geometrical theory of diffraction for an edge in a perfectly conducting surface,' Proc. IEEE 62, 1448-1461 (1974).

${ }^{16} \mathrm{~K}$. B. Rasmussen, "On the effect of terrain profile on sound propagation outdoors," J. Sound Vib. 98, 35-44 (1985).
${ }^{17} \mathrm{~K}$. B. Rasmussen, "Sound propagation over ground under the influence of a sound speed profile in the atmosphere," J. Sound Vib. 139, 71-81 (1990).

${ }^{18}$ K. B. Rasmussen, "Computer simulation of sound propagation over ground under the influence of atmospheric effects. Letter to the editor,' J. Sound Vib. 141, 347-354 (1990).

${ }^{19} \mathrm{~J}$. Kragh and J. Jakobsen, "Propagation of wind turbine noise. Outline of a prediction method," Report AV 320/95, Delta Acoustics and Vibration, Copenhagen, 1995.

${ }^{20}$ W. Lee, N. Bong, W. F. Richards, and R. Raspet, "Impedance formulation of the fast field program for acoustic wave propagation in the atmosphere,', J. Acoust. Soc. Am. 79, 628-634 (1986).

${ }^{21}$ Equation (12) in K. Attenborough, "Ground parameter information for propagation modeling," J. Acoust. Soc. Am. 92, 418-427 (1992).

${ }^{22} \mathrm{~K}$. B. Rasmussen, "The influence of wind on sound propagation over porous ground," Proceedings of 15th International Congress on Acoustics, 26-30 June 1995, Trondheim, Norway, pp. 255-258. 